\title{
Simultaneous acquisition of current and lateral force signals during atomic force microscopy for characterising the piezoelectric and triboelectric effects of ZnO nanorods
}

\section{Yijun Yang}

Seoul National University of Science and Technology

Kwanlae Kim ( $\nabla$ klkim@seoultech.ac.kr)

Seoul National University of Science and Technology

\section{Research Article}

Keywords: AFM, ZnO nanorods, CdS, CdSe

Posted Date: December 1st, 2020

DOl: https://doi.org/10.21203/rs.3.rs-113549/v1

License: (a) (1) This work is licensed under a Creative Commons Attribution 4.0 International License. Read Full License

Version of Record: A version of this preprint was published at Scientific Reports on February 3rd, 2021. See the published version at https://doi.org/10.1038/s41598-021-82506-8. 


\title{
Simultaneous acquisition of current and lateral force signals during atomic force microscopy for characterising the piezoelectric and triboelectric effects of $\mathrm{ZnO}$ nanorods
}

Yijun Yang ${ }^{1}$, Kwanlae Kim ${ }^{1, *}$

${ }^{1}$ Department of Manufacturing Systems and Design Engineering (MSDE), Seoul National University of Science and Technology (SeoulTech), Seoul 01811, Republic of Korea

Corresponding Author: Kwanlae Kim (kkim@seoultech.ac.kr)

\begin{abstract}
Atomic force microscopy (AFM) is central to investigating the piezoelectric potentials of one-dimensional nanomaterials. The AFM probe is used to deflect individual piezoelectric nanorods and to measure the resultant current. However, the torsion data of AFM probes have not been exploited to elucidate the relationship between the applied mechanical force and resultant current. In this study, the effect of the size of $\mathrm{ZnO}$ nanorods on the efficiency of conversion of the applied mechanical force into current was investigated by simultaneously acquiring the conductive AFM and lateral force microscopy signals. The conversion efficiency was calculated based on linear regression analysis of the scatter plot of the data. This method is suitable for determining the conversion efficiencies of all types of freestanding piezoelectric nanomaterials grown under different conditions. A pixel-wise comparison of the current and lateral force images elucidated the mechanism of current
\end{abstract}


generation from dense arrays of $\mathrm{ZnO}$ nanorods. The current signals generated from the $\mathrm{ZnO}$ nanorods by the AFM probe originated from the piezoelectric and triboelectric effects. The current signals contributed by the triboelectric effect were alleviated by using an AFM probe with a smaller spring constant and reducing the normal force.

\section{Introduction}

The piezoelectric effect of $\mathrm{ZnO}$ nanorods was first reported by Wang and Song in $2006{ }^{1}$. Subsequently, an atomic force microscopy (AFM) ${ }^{2,3}$ tip was used to deflect vertically grown $\mathrm{ZnO}$ nanorods at the submicron scale. Because $\mathrm{ZnO}$ exhibits semiconducting and piezoelectric properties ${ }^{4-6}$, the strain induced in the individual $\mathrm{ZnO}$ nanorods by the AFM tip drives a flow of electric charge carriers through the metal-coated AFM tip and $\mathrm{ZnO}$ nanorods. Furthermore, the correlation between the topography signal and conductive atomic force microscopy (C-AFM) ${ }^{7,8}$ signal was analyzed to elucidate the underlying mechanism responsible for the generation of a piezoelectric potential in $\mathrm{ZnO}$ nanorods and to detect the current signal via the AFM tip. Through this experimental study, it was shown that the current signal during C-AFM is detected when the AFM tip touches the compressed side of an $n$-type $\mathrm{ZnO}$ nanorod ${ }^{9}$, as presented in Fig. 1. This comparative analysis of the topography and CAFM signals also helped elucidate the piezoelectric effect in $p$-type $\mathrm{ZnO}$ nanorods ${ }^{10}$ and improved understanding of the effects of the $\mathrm{ZnO}$ nanorod growth method used on the piezoelectric power generation characteristics of the resulting nanorods ${ }^{11}$. C-AFM is used preferentially to investigate the piezoelectricity of one-dimensional nanomaterials with wurtzite structures, such as $\mathrm{CdS}^{12}, \mathrm{CdSe}^{13}, \mathrm{ZnS}^{14}, \mathrm{InN}^{15}$, and $\mathrm{GaN}^{16}$. 
Various types of sensors and energy harvesters based on piezoelectric nanomaterials and with a range of structures have been investigated ${ }^{17-22}$. As these nanomaterials are often subjected to external mechanical forces, understanding their mechanical properties is important. Considering the size of these nanomaterials, lateral force microscopy (LFM) ${ }^{23,24}$ is an effective tool for investigating the elastic moduli of nanomaterials such as $\mathrm{ZnO}^{25,26}, \mathrm{Au}^{27}, \mathrm{Si}$ ${ }^{28}$, and $\mathrm{W}$ nanowires ${ }^{29,30}$. To perform mechanical tests accurately on a single nanowire, AFM scanning is conducted along a programmed manipulation path to deflect the nanowire ${ }^{25,27}$. However, AFM scanning in contact mode also results in the deflection of vertically grown nanorods ${ }^{26}$.

Typically, LFM involves measuring the degree of torsion induced in the AFM cantilever by the surface friction using a position-sensitive photodetector (PSPD) ${ }^{31-33}$. However, when studying the mechanical properties of nanorods, the lateral force applied to the AFM tip may be regarded as the lateral force to which the nanorods are subjected by the AFM tip ${ }^{26}$. Accordingly, by simultaneously monitoring the C-AFM and LFM signals obtained while imaging vertically grown piezoelectric nanorods, the ratio of the output current to the lateral force applied to the $\mathrm{ZnO}$ nanorods can be determined.

We observed the variations in the C-AFM current signal in response to the application of a lateral force to $\mathrm{ZnO}$ nanorods by simultaneously performing C-AFM and LFM. The degree of torsion of an AFM probe can be monitored during AFM operation (including C-AFM) by integrating the AFM instrument with a scanning electron microscope ${ }^{34,35}$. In particular, Wen ${ }^{34}$ suggested that the triboelectric effect and contact potential as well as the piezoelectric effect are responsible for the current signals detected from $\mathrm{ZnO}$ nanorods using an $\mathrm{AFM}$ probe. However, this method requires a sophisticated experimental arrangement. Conversely, 
the C-AFM and LFM signals can be readily acquired simultaneously during AFM operation in contact mode. In this work, this novel experimental method was used to study the effect of the $\mathrm{ZnO}$ nanorod size on the efficiency of conversion of the mechanical force to which they are subjected into current via the piezoelectric and triboelectric effects. Note that if five $\mathrm{ZnO}$ nanorod samples with distinct sizes are deflected by the same AFM tip with a constant normal force, the variation in the lateral force would approximately represent the variation in the net applied mechanical force. Thus, five $\mathrm{ZnO}$ nanorod samples with distinct aspect ratios were prepared, and the ratio of the output current to the mechanical force applied to the nanorods was determined. With respect to AFM-based studies of the piezoelectric effect of $\mathrm{ZnO}$ nanorods, the choice of the AFM probe may have a determining effect on the results, because the normal force in contact mode is determined by the spring constant of the AFM probe. Therefore, the ratio of the output current to the lateral force was determined using two AFM probes with different spring constants. Finally, we attempted to elucidate the mechanism responsible for the generation of current signals in $\mathrm{ZnO}$ nanorods by the AFM tip based on a scatter plot of the current signal versus lateral force and pixel-wise comparison of the C-AFM and LFM images. We confirmed that the piezoelectric and triboelectric effects mainly contribute to the current signals from $\mathrm{ZnO}$ nanorods detected by the AFM tip. Furthermore, the contribution of the triboelectric effect is quite large when an AFM probe with a large spring constant is used with a large normal force.

\section{Results and discussion}

\section{Vertically grown $\mathrm{ZnO}$ nanorods}


The lengths and diameters of the vertically grown $\mathrm{ZnO}$ nanorods, which were measured with a scanning electron microscopy (SEM) system, are shown in Fig. 2. Overall, the aspect ratio of the $\mathrm{ZnO}$ nanorods increases with increasing growth time (see Fig. 2(d)). The details of the nomenclature scheme used for the sample names and growth conditions are given in Methods. SEM images of the five $\mathrm{ZnO}$ nanorod samples are shown in Supplementary Fig. S1.

\section{Representations of current versus lateral force via scatter plot}

As stated previously, the C-AFM and LFM signals were acquired simultaneously as an AFM probe scanned the $\mathrm{ZnO}$ nanorods. Whereas the LFM signal was transmitted through the PSPD, the C-AFM signal was obtained via the current amplifier (Fig. 3). Thus, there was no cross-talk between these two distinct signals. In this study, all the C-AFM and LFM measurements were obtained over a $10 \mu \mathrm{m} \times 5 \mu \mathrm{m}$ area, which resulted in $512 \times 128$ data points. The C-AFM and LFM data points were represented as $c-a f m_{i, j}$ and $l$ f $m_{i, j}$, respectively, using matrix notation. As shown in Fig. 4, a scatter plot was used to represent the correlation between these two sets of data visually. The C-AFM signal from $n$-type $\mathrm{ZnO}$ nanorods has a negative value, whereas the LFM signal during the trace and retrace scans is positive and negative, respectively. Thus, $\left|c-a f m_{i, j}\right|$ and $\left|l f m_{i, j}\right|$ were used for the computations and graphical representations for simplicity.

The C-AFM and LFM measurements were performed on the five samples using two distinct AFM probes. The resultant scatter plots of the current versus lateral force during the trace scans are shown in Fig. 5(a). Both probes (i.e., Probes A and B) were composed of Si and coated with electrically conductive materials, such as Pt, Cr, and Ir, to a thickness of less than 
$30 \mathrm{~nm}$. The actual magnitude of the measured current is dependent on the condition of the metal layer formed on the AFM tip and its degree of wear. Therefore, it was not worth comparing the current levels of the two AFM probes. It can be seen from Fig. 5(a) that regardless of the type of AFM probe used, the points in the scatter plot gradually vary with increasing aspect ratio. For example, in the case of Probe $\mathrm{B}$, when the aspect ratio of the $\mathrm{ZnO}$ nanorods is relatively small, most of the data points lie near the $x$-axis. Thus, although a moderately large lateral force was applied externally to the $\mathrm{ZnO}$ nanorods, only a small current was produced. As the aspect ratio increases, the pattern of the data points changes gradually. In particular, in the S30 scatter plot, the data points for lateral forces corresponding to voltages greater than $7 \mathrm{~V}$ are scarce. Thus, the deflection of sample S30 involved relatively smaller lateral forces than the deflection of the other samples. However, the output current from S30 is noticeably higher that of the other samples. This trend is observed in the scatter plots produced by both Probes A and B. This variation was quantitatively analyzed using the linear regression method, and the corresponding linear model is shown in each scatter plot. The curve fitting tool in Matlab was used to compute the linear model, which can be represented as

$f(x)=p_{1} x+p_{2}$

We also analyzed the effect of the spring constant of the AFM probe on the point pattern. In the scatter plots for $\mathrm{S} 18$, on comparing the number of data points for lateral forces corresponding to voltages greater than $7 \mathrm{~V}$ (marked by dotted boxes), it can be seen that the number of data points decreases when an AFM probe with a higher spring constant is used: Probe A (1496 data points) and Probe B (184 data points). Thus, the degree of torsion of the cantilever is reduced when an AFM probe with a larger spring constant is used to deflect the 
$\mathrm{ZnO}$ nanorods. Furthermore, in the case of Probe $\mathrm{B}$, the data points for lateral forces corresponding to voltages greater than $7 \mathrm{~V}$ are not concentrated near the $x$-axis; in contrast to the case for Probe A.

Such different scatter patterns obtained by Probes A and B can be depicted schematically as shown in Fig. 5(b). If the generation of piezoelectric potential in an $n$-type $\mathrm{ZnO}$ nanorod is solely responsible for the detected current signals, the signals should be detected when an AFM tip touches the compressed side of an $n$-type $\mathrm{ZnO}$ nanorod ${ }^{9}$. If so, the scatter pattern should be similar to the schematic called "S30 by Probe A." However, in the scatter pattern obtained by Probe B, the data points originating from the stretched side are not observed near the lateral force axis. This finding implies that the generation of piezoelectric potential is not only mechanism responsible for the detected current signals. Similar characteristics are also observed in the set of scatter plots corresponding to the retrace scans, as shown in Supplementary Fig. S2. The mechanism underlying the detected current signals from $\mathrm{ZnO}$ nanorods will be elucidated in Fig. 6 .

Interpretation of scatter plots based on pixel-wise comparison of current and lateral

\section{force images}

Although the scatter plots in Fig. 5 present a novel method for assessing the conversion efficiency, they do not intuitively explain the interactions between the AFM tip and $\mathrm{ZnO}$ nanorods. Consequently, we performed local analysis based on pixel-wise comparison of the current and lateral force images. The two pairs of C-AFM and LFM images with $512 \times 128$ data points corresponding to sample S6, which were obtained during the trace scan using 
Probes A and B, are shown in Figs. 6(a) and (b), respectively. (See Supplementary Fig. S3 for the images obtained during the retrace scan.) Each C-AFM and LFM image is marked by rectangles " 1 " and " 2 ," and magnified images corresponding to these marked areas are shown on the right.

It can be seen from Fig. 6 that there is a strong correlation between the C-AFM and LFM images. Furthermore, the degree of correlation is significantly influenced by the type of AFM probe used. On directly comparing the LFM images in Figs. 6(a) and (b), it can be seen that the degree of torsion in the AFM probe is lower for the probe with the larger spring constant (i.e., Probe B). For each pair of local C-AFM and LFM images, the scatter plot of the current versus the lateral force is displayed below the images. These scatter plots of the local images resemble the scatter plot for the complete images in Fig. 5. Furthermore, the proximity of the data points to the $x$-axis was determined by the spring constant of the AFM probe used, as is evident from comparison of the local C-AFM and LFM images. For example, when Probe A was used, some active areas in the LFM image ((a)-1 in Fig. 6(a)) do not correspond to the active areas in the C-AFM image. This discrepancy exists because when Probe A, which had a spring constant of $0.2 \mathrm{~N} / \mathrm{m}$, was used, torsion was occasionally induced in the AFM probe without the deflection of the $\mathrm{ZnO}$ nanorods. Thus, numerous data points lie near the $x$-axis. In contrast, even a small degree of torsion in Probe B, which had a spring constant of $42 \mathrm{~N} / \mathrm{m}$, resulted in the deflection of the $\mathrm{ZnO}$ nanorods and the generation of a current signal. Hence, numerous data points lie some distance from the $x$-axis.

However, the difference in the scatter patterns between Probes A and B shown in Fig. 5(b) is not fully explained yet. Thus, we examined the current and lateral force signals in Figs. 6(a) and (b) more closely. As mentioned earlier, it is known that a current signal is detected when 
an AFM tip touches the compressed side of an $n$-type $\mathrm{ZnO}$ nanorod ${ }^{9}$. This established theory is clearly supported by line profile (a)-1 in Fig. 6(c), which corresponds to Probe A. However, when Probe B was used for imaging, a considerable amount of current signals was detected when the AFM tip was in contact with the stretched side of the $\mathrm{ZnO}$ nanorod (see line profile (b)-1 in Fig. 6(c)). This result is obviously inconsistent with the previously established theory ${ }^{9}$. Recently, it was reported that the current signals detected by an AFM probe originate from the triboelectric effect as well as the piezoelectric effect ${ }^{34}$. Thus, the current signals in line profile (b)-1 can be classified into the region in which the triboelectric effect is dominant (marked by "t") and the region in which both triboelectric and piezoelectric effects simultaneously contribute (marked by " $\mathrm{p}+\mathrm{t}$ "). The current signals contributed by the triboelectric effect are remarkable when the normal force of $420 \mathrm{nN}$ was applied by Probe B. In contrast, the current signals induced by the triboelectric effect are scarcely noticeable in line profile (a)-1 due to the small amount of normal force by Probe $\mathrm{A}^{36}$. The different scatter patterns in Fig. 5(b) are now well explained. Additionally, current signals were detected even after the LFM signals had diminished, as marked by the arrows ("c") in line profiles (a)-1 and (b)-1. This finding implies that current was generated even after the torsion in the probe had been released. It is speculated that these current signals are generated by the contact potential between the AFM probe and $\mathrm{ZnO}$ nanorods ${ }^{34}$.

The variation in the scatter plots for the current versus the lateral force with respect to the aspect ratio of the $\mathrm{ZnO}$ nanorods was obtained from Fig. 5. The gradual variation in the scatter plot in the case of Probe $\mathrm{A}$ is well explained by the results of the pixel-wise comparison of the C-AFM and LFM images, as shown in Fig. 7. All the C-AFM and LFM images were generated based on the color bars shown on the top in Fig. 7. Hence, all 
variations in the C-AFM and LFM signals with changes in the aspect ratio of the $\mathrm{ZnO}$ nanorods could be observed. It can be seen from the magnified images and their corresponding scatter plots that the current increases and the lateral force decreases as the aspect ratio increases. The same analysis results for Probe $\mathrm{B}$ can be found from Supplementary Fig. S4.

\section{Conversion efficiency of applied mechanical force into current via piezoelectric and}

\section{triboelectric effects}

As previously mentioned, when C-AFM and LFM are performed on $\mathrm{ZnO}$ nanorods, the net external force comprises the normal and lateral forces. For each AFM probe, the measurements were obtained in contact mode under a constant normal force corresponding to the setpoint of the feedback circuit. This approach allowed for the following approximation of the conversion efficiency:

$\frac{\text { Total output current }}{\text { Total input mechanical force }} \approx \alpha \frac{\sum_{i=1, j=1}^{i=128, j=512}\left|c-a f m_{i, j}\right|}{\sum_{i=1, j=1}^{i=128, j=512}\left|l f m_{i, j}\right|}$,

where $\alpha$ is a scaling factor that considers the setpoint level and electrical conductivity of the AFM tip. The effect of $\alpha$ on the conversion efficiency became almost negligible when the same AFM probe was used for all the five samples (S6-S30) along with a constant setpoint. Note that there was no noticeable wear of the AFM tip during the measurements of the five samples. Therefore, the conversion efficiency was computed for the five samples for each AFM probe during both the trace and retrace scans.

As shown in Fig. 8(a), the conversion efficiency computed from the trace and retrace scan 
data sets increases with increasing aspect ratio. The conversion efficiency is, simply put, the ratio of the total current to the total lateral force. Thus, in the cases in which the data points are concentrated near the $y$-axis, the conversion efficiency is large. The pattern of the data points could be effectively described by the slope of the linear model $\left(p_{1}\right)$ in Eq. (1). The computed $p_{1}$ values for the five samples and two AFM probes are shown in Fig. 8(b). Overall, $p_{1}$ also increases as the aspect ratio increases. The results in Fig. 8(b) suggest that a larger amount of current is generated by the $\mathrm{ZnO}$ nanorods for a smaller external mechanical force as the aspect ratio of the nanorods increases. Therefore, the results of the linear regression analysis agree with those of the conversion efficiency calculations performed using Eq. (2).

As can be seen in Fig. 6(c), the current signals originating from the triboelectric effect are significant when a $420 \mathrm{nN}$ normal force is applied by Probe B. The measured current signals contributed by the triboelectric effect are effectively reduced with a $30 \mathrm{nN}$ normal force by Probe A. The total lateral force, $l_{\text {tot }}\left(=\sum_{i=1, j=1}^{i=128, j=512}\left|l \mathrm{fm}_{i, j}\right|\right)$, from Probe A was computed for the five samples and was found to decrease with increasing aspect ratio (Supplementary Fig. S5). This finding implies that, for Probe A, the increased conversion efficiency with increasing aspect ratio in Fig. 8(a) is not induced by enhanced triboelectric effect. Therefore, for Probe A, the enhanced piezoelectric potential with increasing aspect ratio of the $\mathrm{ZnO}$ nanorod is responsible for the increased conversion efficiency in Fig. 8(a) and the increase in $p_{1}$ in Fig. 8(b). Likewise, $l_{\text {tot }}$ for Probe $\mathrm{B}$ does not show any increasing or decreasing trend with increasing aspect ratio of the $\mathrm{ZnO}$ nanorod. Thus, it can be concluded that although the effects of triboelectricity on the measured current signals are considerable, the increases in the current signals with increasing aspect ratio were not induced by triboelectric effect. 
The experimental results shown in Figs. 5 and 8 for Probe A agree with those of previous works on the effect of the $\mathrm{ZnO}$ nanorod size on piezoelectricity ${ }^{11,37,38}$. It has been reported that the electrical energy increases as the aspect ratio of a $\mathrm{ZnO}$ nanorod increases through finite element simulations ${ }^{11,38}$ and experimental work ${ }^{37}$. This behavior was explained by two factors. First, the amount of deflection increases with increasing aspect ratio of the $\mathrm{ZnO}$ nanorod. Second, the free carrier concentration induced by crystallographic defects decreases as the aspect ratio increases, enhancing the piezoelectric effect. In the present study, the output current increased and the actual mechanical force deflecting the $\mathrm{ZnO}$ nanorods decreased as the aspect ratio of the $\mathrm{ZnO}$ nanorods increased.

In general, the sizes and structures of freestanding piezoelectric nanomaterials are determined by the growth method and conditions used. The experimental and analysis methods proposed in this study enable one to assess readily the efficiency of conversion of the mechanical force applied to nanomaterials into current. Thus, these methods should facilitate the optimization of the synthesis of nanomaterials for use in piezoelectric sensors and energy harvesters. Additionally, it is important to use an AFM probe with a small spring constant to minimize the influence of the triboelectric effect on the results.

\section{Conclusions}

In this study, we performed comprehensive analysis of the piezoelectric and triboelectric effects generated in $\mathrm{ZnO}$ nanorods using an AFM probe. By simultaneously acquiring the CAFM and LFM signals under a constant normal force, the variations in the ratio of the output current to the applied mechanical force with changes in the aspect ratio of the $\mathrm{ZnO}$ nanorods 
could be observed. In particular, two AFM probes with spring constants of 0.2 and $42.0 \mathrm{~N} / \mathrm{m}$ were used to investigate the effects of the spring constant on the analysis results. The efficiency of the conversion of the lateral force into the output current was computed, and it was found that the efficiency was enhanced with increasing aspect ratio of the $\mathrm{ZnO}$ nanorods. In addition, scatter plots of the current versus the lateral force were produced, and the variations in the patterns of the data points with changes in the aspect ratio were analyzed quantitatively using linear regression. The resulting linear model showed that the slope increased with increasing aspect ratio. This finding indicates that there is a transition of the data points in the scatter plots from the lateral force axis ( $x$-axis) towards the current axis $(y$ axis) as the aspect ratio increases. The variations in the scatter patterns with changes in the aspect ratio of the $\mathrm{ZnO}$ nanorods agreed with the results of a pixel-wise comparison of the $\mathrm{C}$ AFM and LFM images. It was confirmed that the current signals were contributed by both the piezoelectric and triboelectric effects in a $\mathrm{ZnO}$ nanorod. The effect of triboelectricity on the measured current signals was alleviated by reducing a normal force and the spring constant of an AFM probe. This analysis method is expected to be applicable to all freestanding piezoelectric nanomaterials. Furthermore, it is anticipated to facilitate analysis of the effects of the growth conditions of nanomaterials on the efficiency of conversion of the applied mechanical force into the output current.

\section{Methods}

\section{Growth of ZnO nanorods}

The $\mathrm{ZnO}$ nanorods used in this study were synthesized on $p$-Si (100) substrates via a two-step 
hydrothermal method ${ }^{39,40}$. The $\mathrm{Si}$ substrates were sonicated in acetone, ethanol, and deionized water for $10 \mathrm{~min}$ each. The seed layer was grown in $50 \mathrm{mM}$ zinc acetate dihydrate $\left(\mathrm{Zn}\left(\mathrm{Ch}_{3} \mathrm{COO}\right)_{2} \cdot 2 \mathrm{H}_{2} \mathrm{O}\right)$, dissolved in ethanol at $80{ }^{\circ} \mathrm{C}$ for $30 \mathrm{~s}$, and then dried on a hot plate at $100{ }^{\circ} \mathrm{C}$ for $5 \mathrm{~min}$. This process was repeated three times. The $\mathrm{ZnO}$ nanorods were grown on top of the seed layer using a precursor solution that was a mixture of $50 \mathrm{mM}$ zinc nitrate hexahydrate $\left(\mathrm{Zn}\left(\mathrm{NO}_{3}\right)_{2} \cdot 6 \mathrm{H}_{2} \mathrm{O}\right)$ and hexamethylenetetramine $\left(\left(\mathrm{CH}_{2}\right)_{6} \mathrm{~N}_{4}\right)$ dissolved in deionized water at $80{ }^{\circ} \mathrm{C}$. Five nanorod samples with distinct aspect ratios were prepared by varying the growth time (Table 1). The lengths and diameters of the $\mathrm{ZnO}$ nanorods were measured from their field emission scanning electron microscopy (JSM6700F, JEOL) images.

\section{Conductive atomic force microscopy and lateral force microscopy}

The C-AFM and LFM measurements were obtained using an AFM instrument (XE-150, Park Systems). A C-AFM module with a current amplifier was installed in the AFM system. In this work, two types of AFM probes were used, as listed in Table 2. The spring constants of these AFM probes were 0.2 and $42.0 \mathrm{~N} / \mathrm{m}$. The appropriate set point for each AFM probe is approximately 10 times the spring constant for stable AFM operation in contact mode. However, the spring constant of Probe A was too small to deflect the $\mathrm{ZnO}$ nanorods, and, therefore, a set point of $30 \mathrm{nN}$ was applied. Table 2 lists the conditions for the AFM scans performed using the two probes. During all the AFM measurements, the cantilever was consistently oriented perpendicular to the fast-scan direction.

\section{Data availability}


The datasets generated during and/or analysed during the current study are available from the corresponding author on reasonable request. 


\section{References}

1. Wang, Z. L. \& Song, J. Piezoelectric nanogenerators based on zinc oxide nanowire arrays. Science 312, 242-246 (2006). https://doi.org/10.1126/science.1124005

2. Giessibl, F. J. Advances in atomic force microscopy. Rev. Mod. Phys. 75, 949-983 (2003). https://doi.org/10.1103/RevModPhys.75.949

3. Binnig, G., Quate, C. F. \& Gerber, C. Atomic force microscope. Phys. Rev. Lett. 56, 930-933 (1986). https://doi.org/10.1103/PhysRevLett.56.930

4. Wang, Z. L. Zinc oxide nanostructures: growth, properties and applications. J. Phys. Condens. Matter. 16, R829-R858 (2004). https://doi.org/10.1088/0953-8984/16/25/R01

5. Schmidt-Mende, L. \& MacManus-Driscoll, J. L. ZnO - nanostructures, defects, and devices. Mater. Today 10, 40-48 (2007). https://doi.org/10.1016/S1369-7021(07)70078-0

6. Özgür, Ü. et al. A comprehensive review of $\mathrm{ZnO}$ materials and devices. J. Appl. Phys. 98, 041301 (2005). https://doi.org/10.1063/1.1992666

7. Pomarico, A. A. et al. Current mapping of GaN films by conductive atomic force microscopy. Appl. Phys. Lett. 82, 1890-1892 (2003). https://doi.org/10.1063/1.1563054

8. Alexeev, A., Loos, J. \& Koetse, M. M. Nanoscale electrical characterization of semiconducting polymer blends by conductive atomic force microscopy (C-AFM). Ultramicroscopy 106, 191-199 (2006). https://doi.org/10.1016/j.ultramic.2005.07.003

9. Song, J., Zhou, J. \& Wang, Z. L. Piezoelectric and semiconducting coupled power generating process of a single $\mathrm{ZnO}$ belt/wire. A technology for harvesting electricity from the environment. Nano Lett. 6, 1656-1662 (2006). https://doi.org/10.1021/n1060820v

10. Lu, M. P. et al. Piezoelectric nanogenerator using p-type $\mathrm{ZnO}$ nanowire arrays. Nano Lett. 9, 1223-1227 (2009). https://doi.org/10.1021/n1900115y

11. Riaz, M., Song, J., Nur, O., Wang, Z. L. \& Willander, M. Study of the piezoelectric power generation of $\mathrm{ZnO}$ nanowire arrays grown by different methods. Adv. Funct. Mater. 21, 628-633 (2011). https://doi.org/10.1002/adfm.201001203

12. Lin, Y. F., Song, J., Ding, Y., Lu, S. Y. \& Wang, Z. L. Piezoelectric nanogenerator using CdS nanowires. Appl. Phys. Lett. 92, 022105 (2008). https://doi.org/10.1063/1.2831901

13. Zhou, Y. S. et al. Vertically aligned CdSe nanowire arrays for energy harvesting and piezotronic devices. ACS Nano 6, 6478-6482 (2012). https://doi.org/10.1021/nn3022074

14. Lu, M. Y. et al. $\mathrm{ZnO}-\mathrm{ZnS}$ heterojunction and $\mathrm{ZnS}$ nanowire arrays for electricity generation. ACS Nano 3, 357-362 (2009). https://doi.org/10.1021/nn800804r

15. Huang, C. T. et al. Single-InN-nanowire nanogenerator with upto $1 \mathrm{v}$ output voltage. Adv. Mater. 22, 4008-4013 (2010). https://doi.org/10.1002/adma.201000981 
16. Chen, C. Y. et al. Gallium nitride nanowire based nanogenerators and light-emitting diodes. ACS Nano 6, 5687-5692 (2012). https://doi.org/10.1021/nn301814w

17. Xu, S. et al. Self-powered nanowire devices. Nat. Nanotechnol. 5, 366-373 (2010). https://doi.org/10.1038/nnano.2010.46

18. $\mathrm{Wu}, \mathrm{W}$. et al. Lead zirconate titanate nanowire textile nanogenerator for wearable energy-harvesting and self-powered devices. ACS Nano 6, 6231-6235 (2012). https://doi.org/10.1021/nn3016585

19. Koka, A. \& Sodano, H. A. High-sensitivity accelerometer composed of ultra-long vertically aligned barium titanate nanowire arrays. Nat. Commun. 4, 2682 (2013). https://doi.org/10.1038/ncomms3682

20. Fan, F. R., Tang, W. \& Wang, Z. L. Flexible nanogenerators for energy harvesting and self-powered electronics. Adv. Mater. 28, 4283-4305 (2016). https://doi.org/10.1002/adma.201504299

21. Wang, Z. L., Zhu, G., Yang, Y., Wang, S. \& Pan, C. Progress in nanogenerators for portable electronics. Mater. Today 15, 532-543 (2012). https://doi.org/10.1016/S13697021(13)70011-7

22. Koka, A., Zhou, Z. \& Sodano, H. A. Vertically aligned BaTiO3 nanowire arrays for energy harvesting. Energy Environ. Sci. 7, 288-296 (2014). https://doi.org/10.1039/c3ee42540a

23. Bhushan, B. Nanotribology and nanomechanics. Wear 259, 1507-1531 (2005). https://doi.org/10.1016/j.wear.2005.01.010

24. Munz, M. Force calibration in lateral force microscopy: a review of the experimental methods. J. Phys. D. Appl. Phys. 43, 063001 (2010). https://doi.org/10.1088/0022$3727 / 43 / 6 / 063001$

25. Wen, B., Sader, J. E. \& Boland, J. J. Mechanical properties of ZnO nanowires. Phys. Rev. Lett. 101, 175502 (2008). https://doi.org/10.1103/PhysRevLett.101.175502

26. Song, J., Wang, X., Riedo, E. \& Wang, Z. L. Elastic property of vertically aligned nanowires. Nano Lett. 5, 1954-1958 (2005). https://doi.org/10.1021/nl051334v

27. Wu, B., Heidelberg, A. \& Boland, J. J. Mechanical properties of ultrahigh-strength gold nanowires. Nat. Mater. 4, 525-529 (2005). https://doi.org/10.1038/nmat1403

28. Heidelberg, A. et al. A generalized description of the elastic properties of nanowires. Nano Lett. 6, 1101-1106 (2006). https://doi.org/10.1021/nl060028u

29. Röhlig, C. C. et al. Elastic properties of nanowires. Phys. Status Solidi B 247, 25572570 (2010). https://doi.org/10.1002/pssb.201046378

30. Cimalla, V. et al. Nanomechanics of single crystalline tungsten nanowires. $J$. Nanomater. 2008, 638947 (2008). https://doi.org/10.1155/2008/638947 
31. Marsden, A. J., Phillips, M. \& Wilson, N. R. Friction force microscopy: a simple technique for identifying graphene on rough substrates and mapping the orientation of graphene grains on copper. Nanotechnology 24, 255704 (2013). https://doi.org/10.1088/0957$4484 / 24 / 25 / 255704$

32. Lin, L. Y., Kim, D. E., Kim, W. K. \& Jun, S. C. Friction and wear characteristics of multi-layer graphene films investigated by atomic force microscopy. Surf. Coat. Tech. 205, 4864-4869 (2011). https://doi.org/10.1016/j.surfcoat.2011.04.092

33. Filleter, T. \& Bennewitz, R. Structural and frictional properties of graphene films on $\mathrm{SiC}(0001)$ studied by atomic force microscopy. Phys. Rev. B 81, 155412 (2010). https://doi.org/10.1103/PhysRevB.81.155412

34. Wen, C. et al. In situ observation of current generation in $\mathrm{ZnO}$ nanowire based nanogenerators using a CAFM Integrated into an SEM. ACS Appl. Mater. Interfaces 11, 15183-15188 (2019). https://doi.org/10.1021/acsami.9b00447

35. Hoffmann, S. et al. Measurement of the bending strength of vapor-liquid-solid grown silicon nanowires. Nano Lett. 6, 622-625 (2006). https://doi.org/10.1021/n1052223z

36. Pan, S., Yin, N. \& Zhang, Z. Time-\& load-dependence of triboelectric effect. Sci. Rep. 8, 2470 (2018). https://doi.org/10.1038/s41598-018-20937-6

37. Rivera, V. F. et al. Length-dependent charge generation from vertical arrays of highaspect-ratio $\mathrm{ZnO}$ nanowires. Chem. Eur. J. 19, 14665-14674 (2013). https://doi.org/10.1002/chem.201204429

38. Hinchet, R. et al. Performance optimization of vertical nanowire-based piezoelectric nanogenerators. $\quad A d v$. Funct. $\quad$ Mater. $\quad \mathbf{2 4}, \quad 971-977 \quad$ (2014). https://doi.org/10.1002/adfm.201302157

39. Wang, S. F., Tseng, T. Y., Wang, Y. R., Wang, C. Y. \& Lu, H. C. Effect of ZnO seed layers on the solution chemical growth of $\mathrm{ZnO}$ nanorod arrays. Ceram. Int. 35, 1255-1260 (2009). https://doi.org/10.1016/j.ceramint.2008.06.012

40. Lee, Y. S., Lee, S. N. \& Park, I. K. Growth of $\mathrm{ZnO}$ hemispheres on silicon by a hydrothermal method. Ceram. Int. 39, 3043-3048 (2013). https://doi.org/10.1016/j.ceramint.2012.09.083 


\section{Acknowledgements}

This work was supported by the National Research Foundation of Korea(NRF) grant funded by the Korea government(MSIT) (2019R1F1A1057944). We would like to thank Prof. Il-Kyu Park at Seoul National University of Science and Technology for his help with growing ZnO nanorods.

\section{Author contributions statement}

Y.Y. grew and characterised $\mathrm{ZnO}$ nanorod samples including C-AFM and LFM measurements. Y.Y. and K.K. carried out data analysis. K.K., who supervised this research, conceived the idea for the simultaneous acquisition of C-AFM and LFM signals for $\mathrm{ZnO}$ nanorods. Y.Y. and K.K. prepared the manuscript.

\section{Additional information}

\section{Competing interests}

The authors declare no competing interests. 
Fig. 1. Piezoelectric potential induced by the AFM probe. (a) Generation of piezoelectric potential by deflection of the $\mathrm{ZnO}$ nanorod, (b) current passing through the metal-coated AFM tip in contact with the $\mathrm{ZnO}$ nanorod, (c) topography and C-AFM signals during the process in (a). The current signals originating from the piezoelectric potential are detected when the AFM tip touches the compressed side of an n-type $\mathrm{ZnO}$ nanorod ${ }^{1,9}$.

Fig. 2. Growth of $\mathrm{ZnO}$ nanorod samples. (a) Top view and (b) side view of vertically grown $\mathrm{ZnO}$ nanorods (S18) and (c) lengths and diameters and (d) aspect ratios of five $\mathrm{ZnO}$ nanorod samples (S6-S30).

Fig. 3. Schematic of the experimental setup. Simultaneous acquisition of current and lateral force signals during the scan of vertically grown $\mathrm{ZnO}$ nanorods using an AFM probe.

Fig. 4. Scatter plot of current and lateral force signals. During a typical C-AFM measurement, a constant normal force was applied to the $\mathrm{ZnO}$ nanorods. The magnitude of the lateral force applied to the nanorods changed continuously.

Fig. 5. Effects of $\mathrm{ZnO}$ nanorod size on the ratio of the current to the lateral force. (a) Scatter plots of current versus lateral force signals for five samples, as measured by Probes A and B during trace scans. (b) Schematics illustrating the different scatter patterns between Probes A and B for S30.

Fig. 6. Pixel-wise comparison of the current and lateral force images. Pairs of current and lateral force images obtained using (a) Probe A and (b) Probe B during trace scans. Magnified images of the small areas marked by boxes are provided, with corresponding scatter plots on the right. (c) Line profiles of the magnified current and lateral force images in (a) and (b). (d) 
Schematics of three distinct current generation mechanisms: contact potential, triboelectric effect, and simultaneous piezoelectric and triboelectric effects ${ }^{34}$.

Fig. 7. Variations in current and lateral force signals with the size of the $\mathrm{ZnO}$ nanorods. Pairs of current and lateral force images for (a) S6, (b) S12, (c) S18, (d) S24, and (e) S30 as measured by Probe A. In each figure part, magnified images of the areas marked by boxes and corresponding scatter plots are shown on the right. All images were obtained during trace scans.

Fig. 8. Efficiency of conversion of the lateral force applied to the $\mathrm{ZnO}$ nanorods into current depending on the $\mathrm{ZnO}$ nanorod size. (a) Conversion efficiencies (total current/total lateral force) computed for each sample during trace and retrace scans using Probes A and B. (b) $p_{1}$ values for linear models (Eq. (1)) computed for each sample during trace and retrace scans by Probes A and B. 


\begin{tabular}{|c|c|c|c|}
\hline Sample name & $\begin{array}{c}\text { Precursor } \\
\text { concentration }\end{array}$ & Temperature & Growth time $(\mathrm{h})$ \\
\hline S6 & & & 6 \\
\hline S12 & & & 12 \\
\hline S18 & $50 \mathrm{mM}$ & $80^{\circ} \mathrm{C}$ & 18 \\
\hline S24 & & & 24 \\
\hline S30 & & & 30 \\
\hline
\end{tabular}

Table 1. Growth conditions for $\mathrm{ZnO}$ nanorod samples.

\begin{tabular}{lcccc}
\hline & AFM probes & $\begin{array}{c}\text { Spring constant } \\
(\mathrm{N} / \mathrm{m})\end{array}$ & $\begin{array}{c}\text { Set point } \\
(\mathrm{nN})\end{array}$ & $\begin{array}{c}\text { Scan speed } \\
\end{array}$ \\
& & & & \\
\hline Probe A & ElectriCont-G & 0.2 & 30 & 0.2 \\
\hline Probe B & PPP-NCHPt & 42.0 & 420 & 0.2 \\
\hline
\end{tabular}

Table 2. AFM scan conditions for the two AFM probes used. 
Figures

(a)

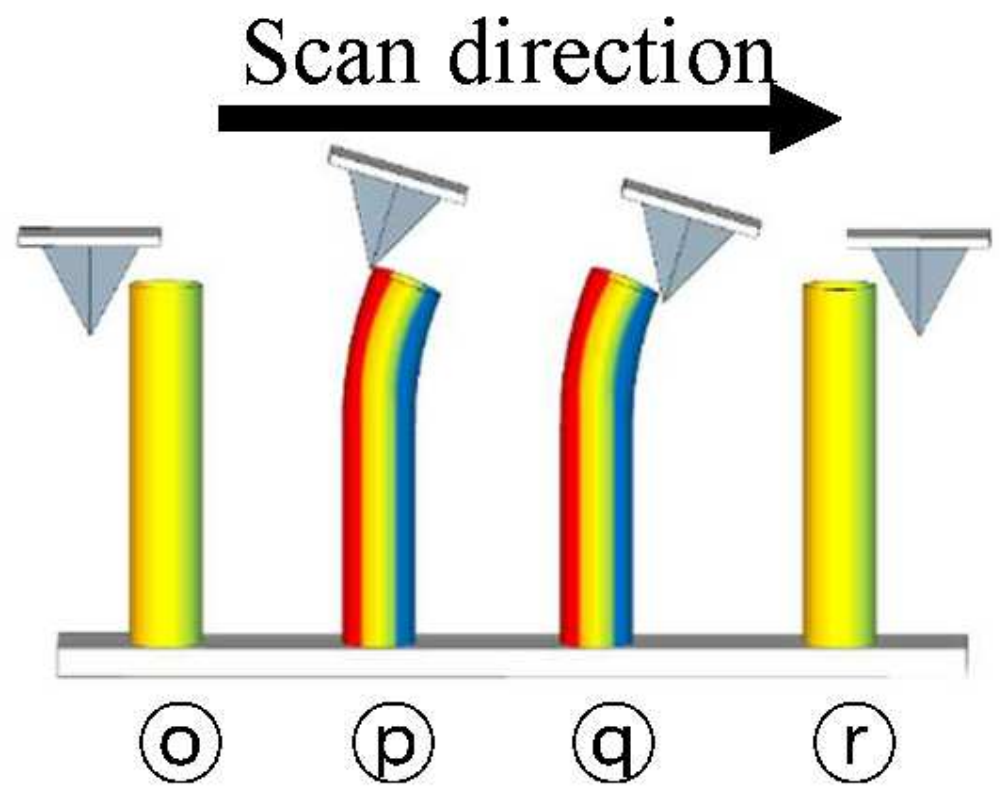

(D) (p) (r)

(c)

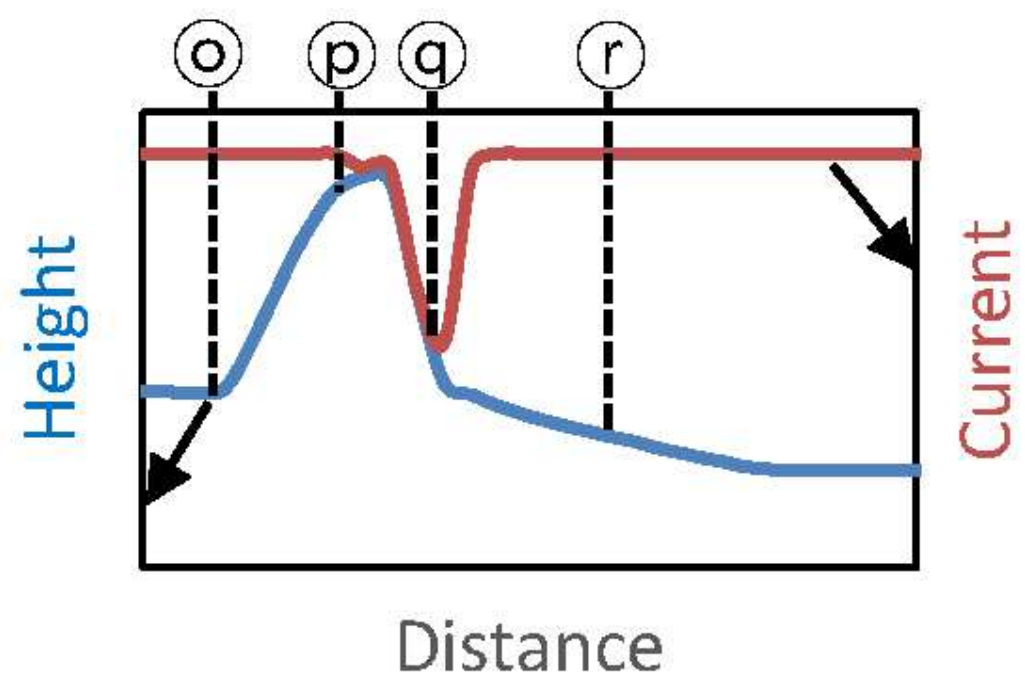

(b)

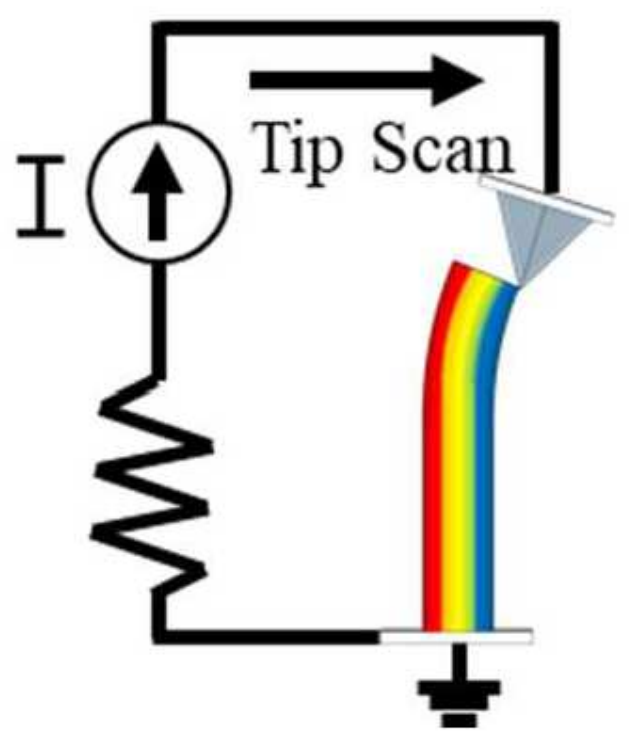

Figure 1

Piezoelectric potential induced by the AFM probe. (a) Generation of piezoelectric potential by deflection of the $\mathrm{ZnO}$ nanorod, (b) current passing through the metal-coated AFM tip in contact with the $\mathrm{ZnO}$ nanorod, (c) topography and C-AFM signals during the process in (a). The current signals originating from the 
piezoelectric potential are detected when the AFM tip touches the compressed side of an n-type ZnO nanorod 1,9.
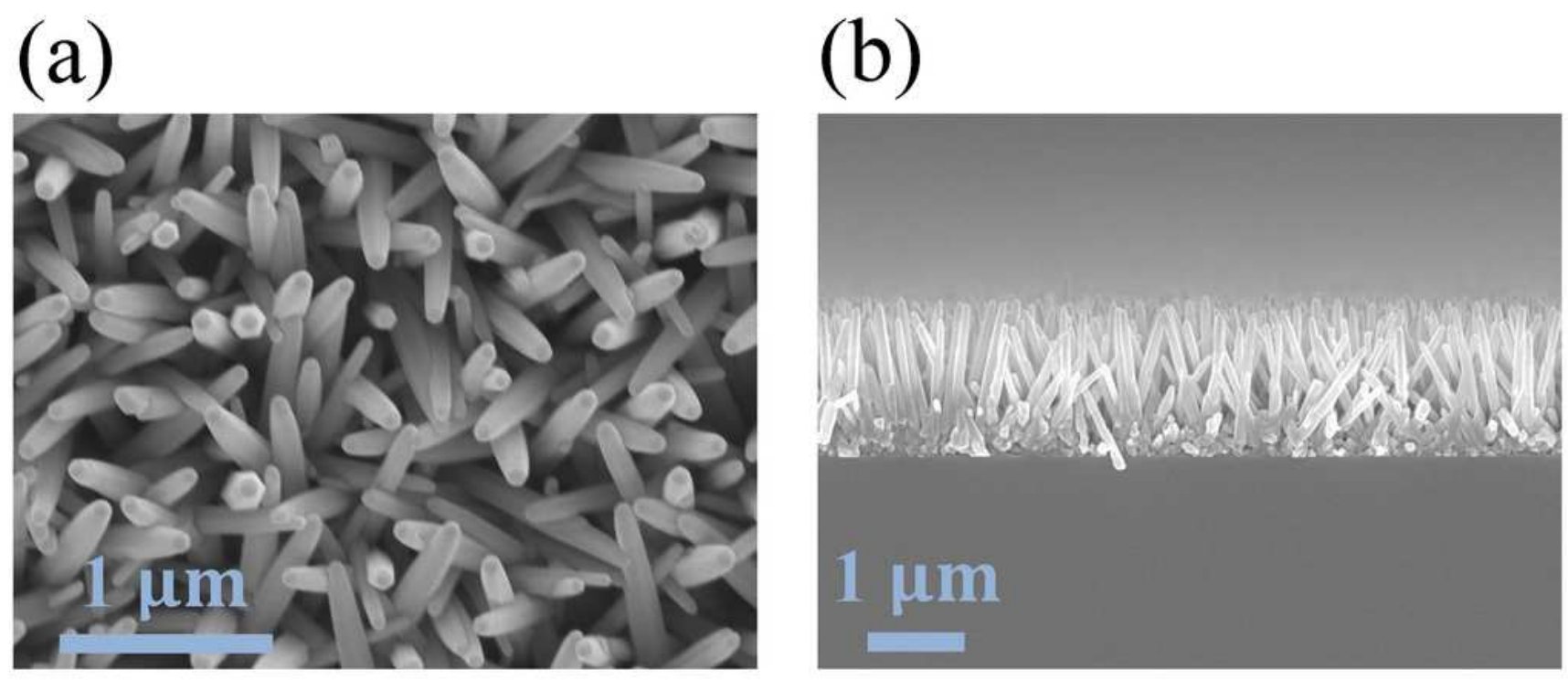

\section{(c)}

(d)
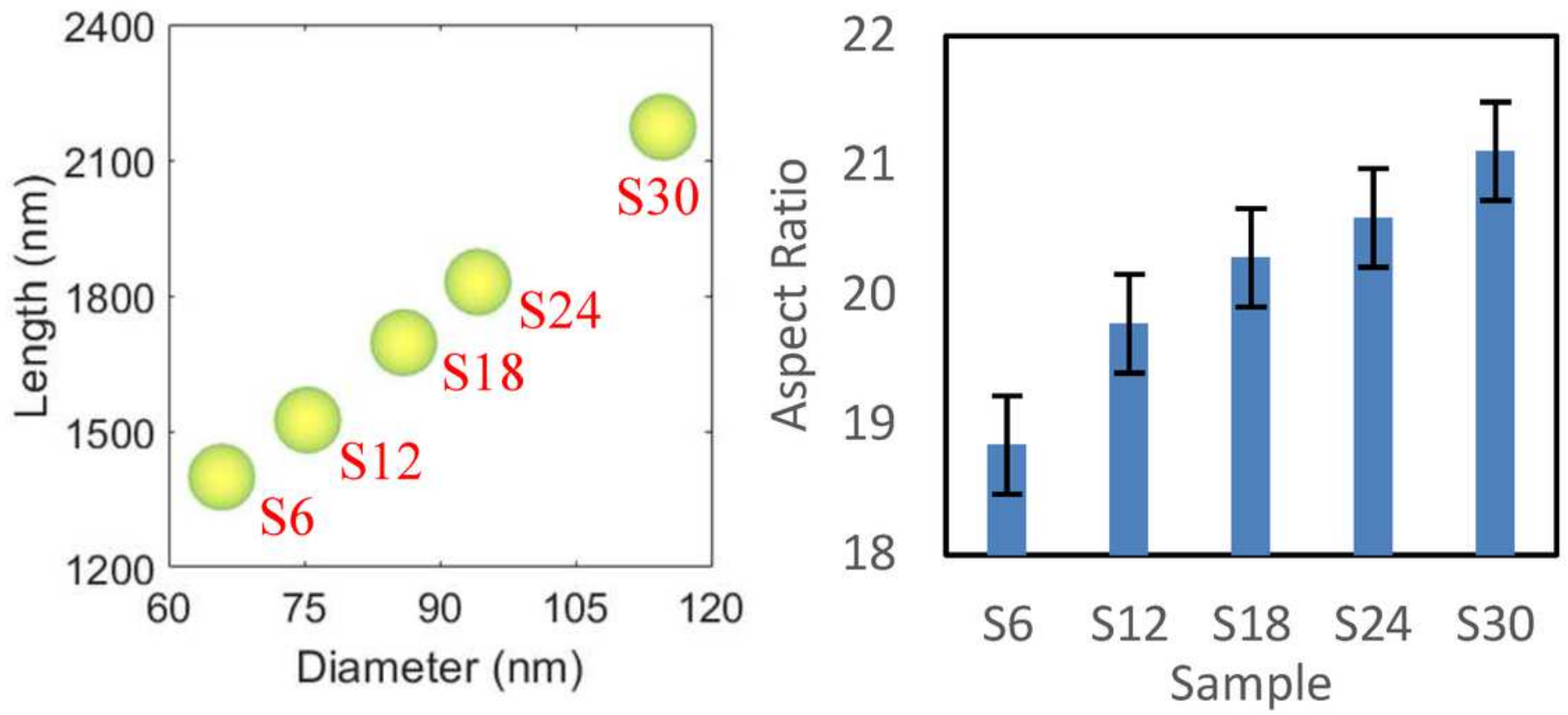

Figure 2

Growth of ZnO nanorod samples. (a) Top view and (b) side view of vertically grown ZnO nanorods (S18) and (c) lengths and diameters and (d) aspect ratios of five ZnO nanorod samples (S6खS30). 


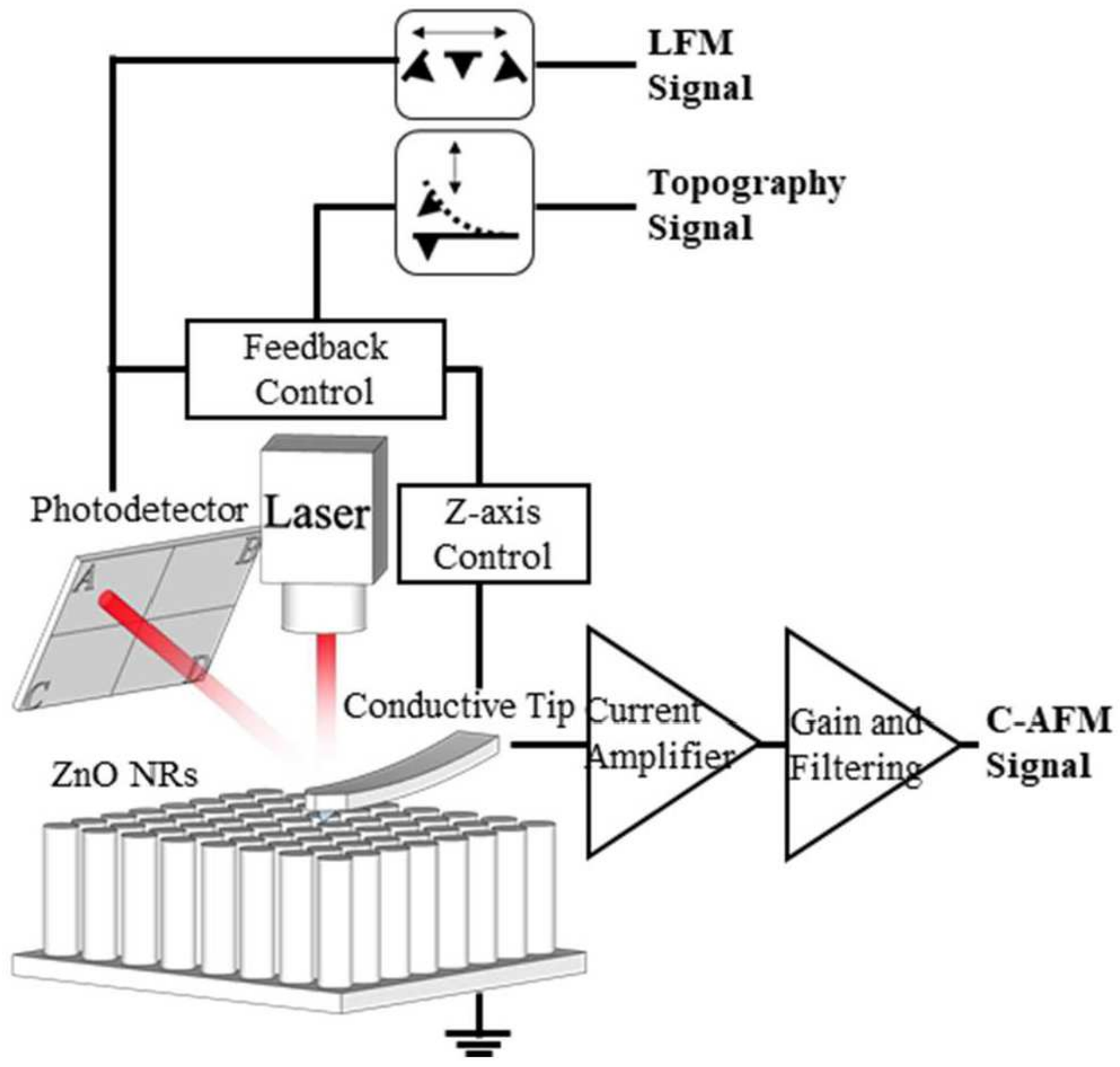

Figure 3

Scatter plot of current and lateral force signals. During a typical C-AFM measurement, a constant normal force was applied to the $\mathrm{ZnO}$ nanorods. The magnitude of the lateral force applied to the nanorods changed continuously. 


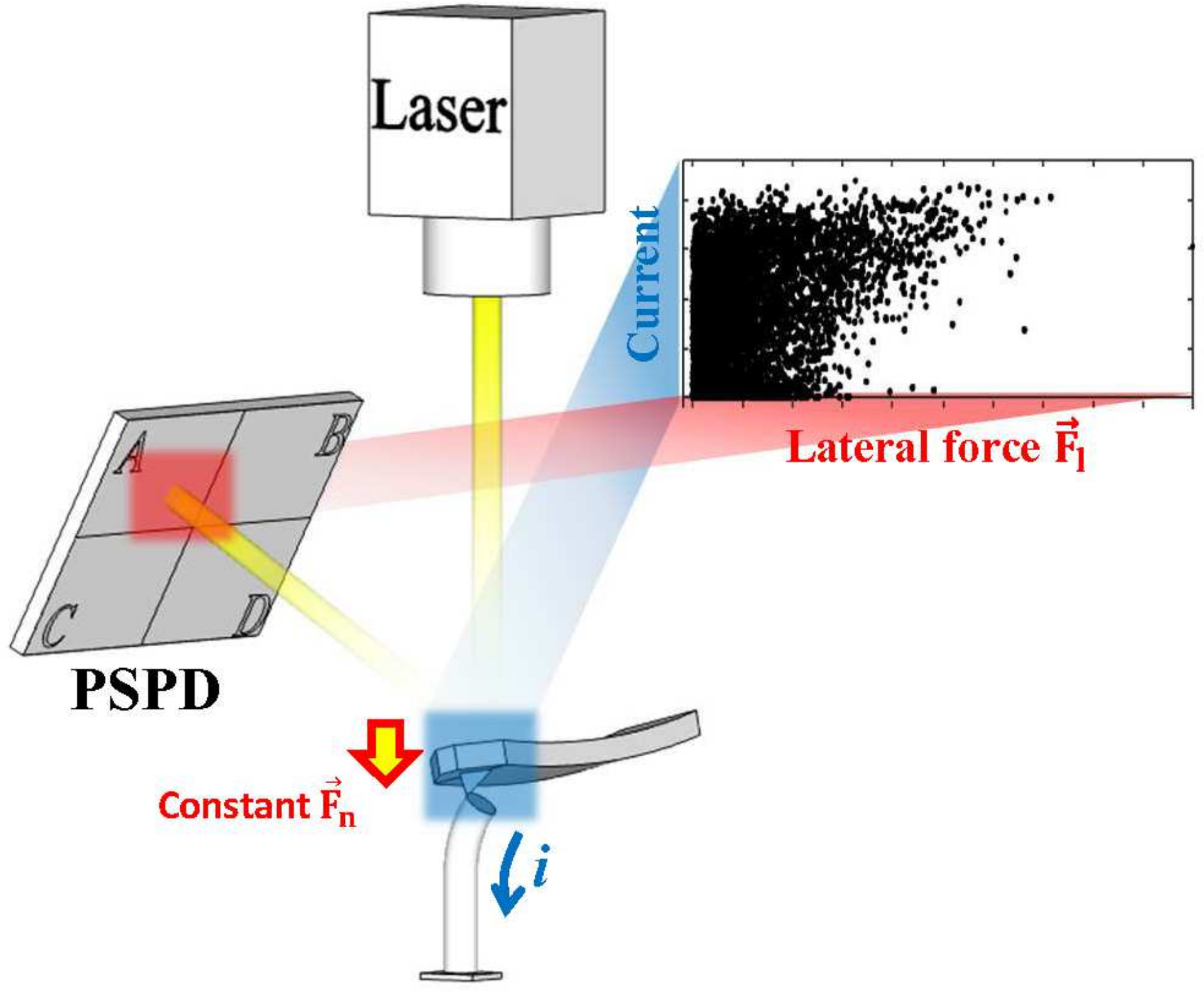

Figure 4

Effects of $\mathrm{ZnO}$ nanorod size on the ratio of the current to the lateral force. (a) Scatter plots of current versus lateral force signals for five samples, as measured by Probes A and B during trace scans. (b) Schematics illustrating the different scatter patterns between Probes A and B for S30. 
(a)

S6
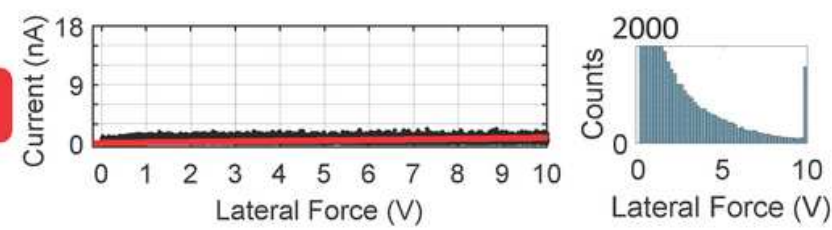

S12
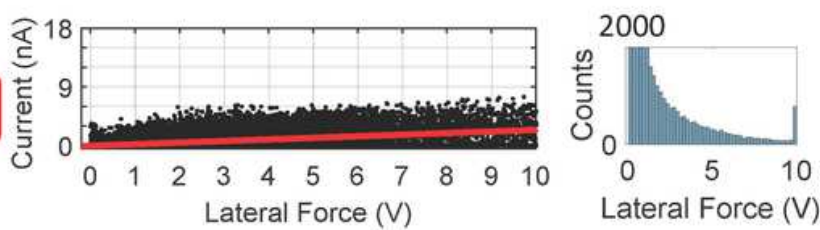

S18
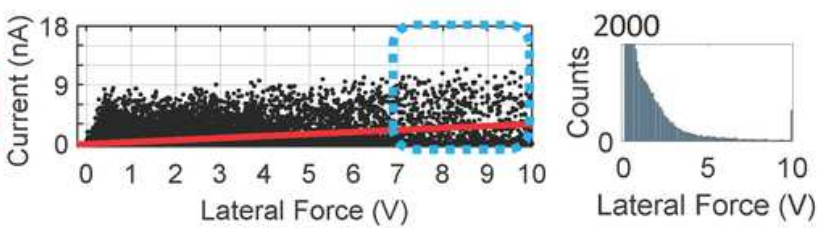

S24
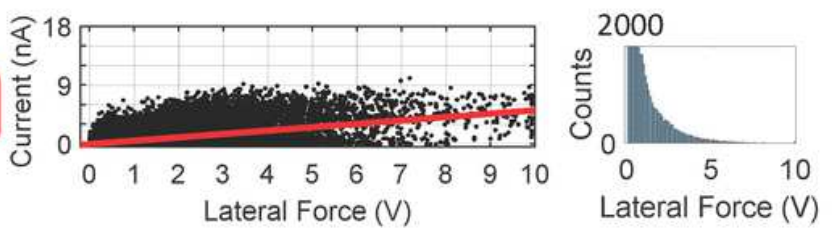

S30
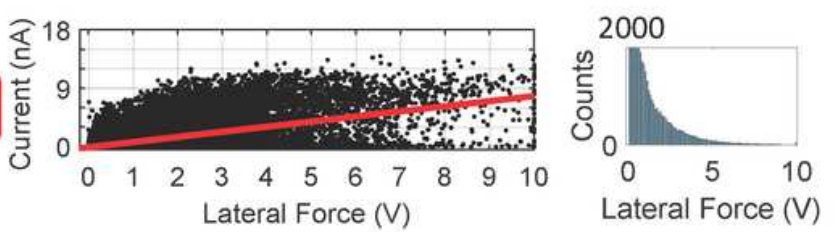

(b)

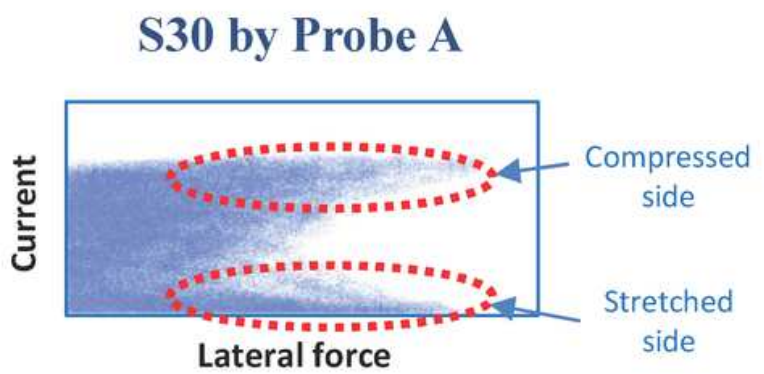

Probe B
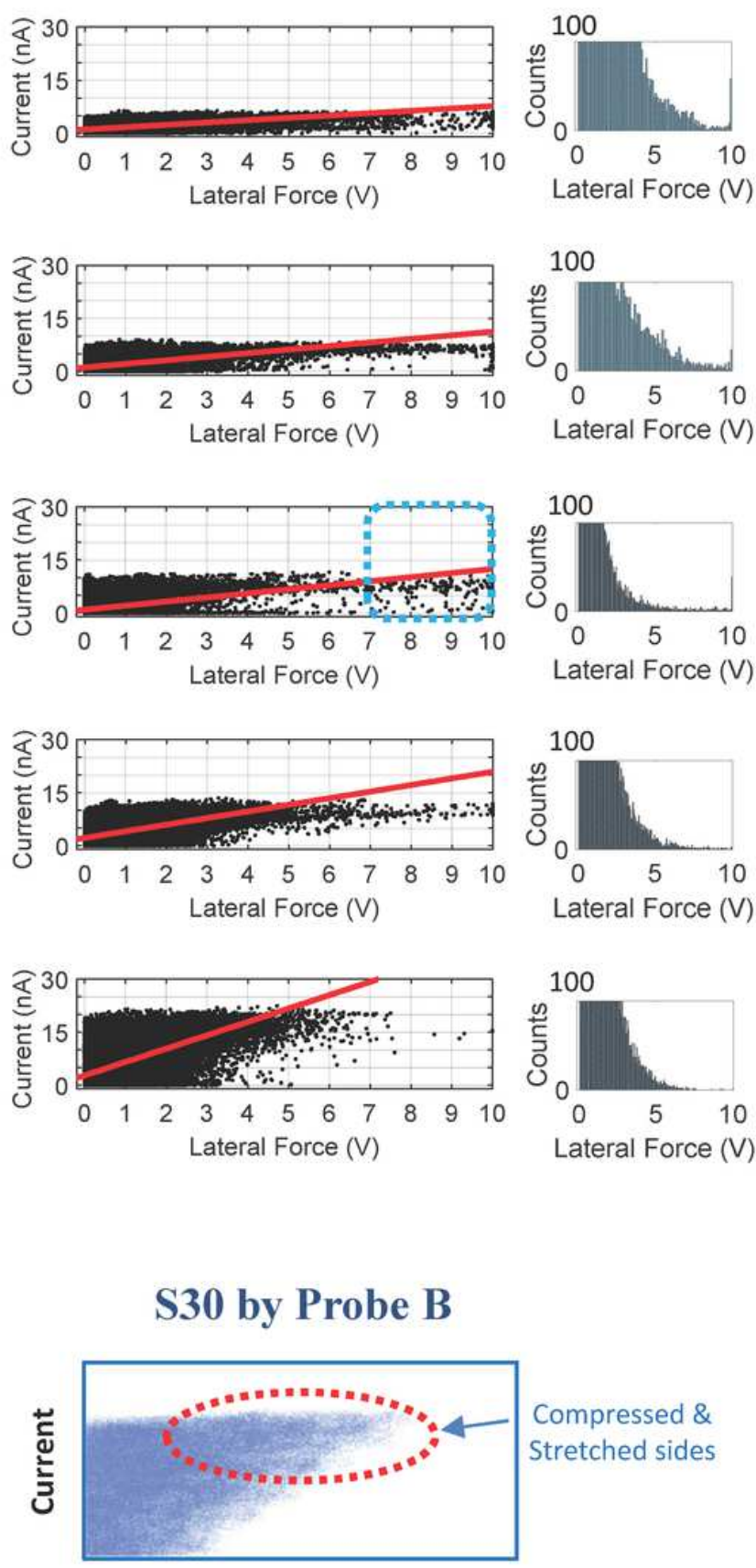

Lateral force

Figure 5

Effects of $\mathrm{ZnO}$ nanorod size on the ratio of the current to the lateral force. (a) Scatter plots of current versus lateral force signals for five samples, as measured by Probes A and B during trace scans. (b) Schematics illustrating the different scatter patterns between Probes A and B for S30. 
(a)

\section{Fast scan direction}

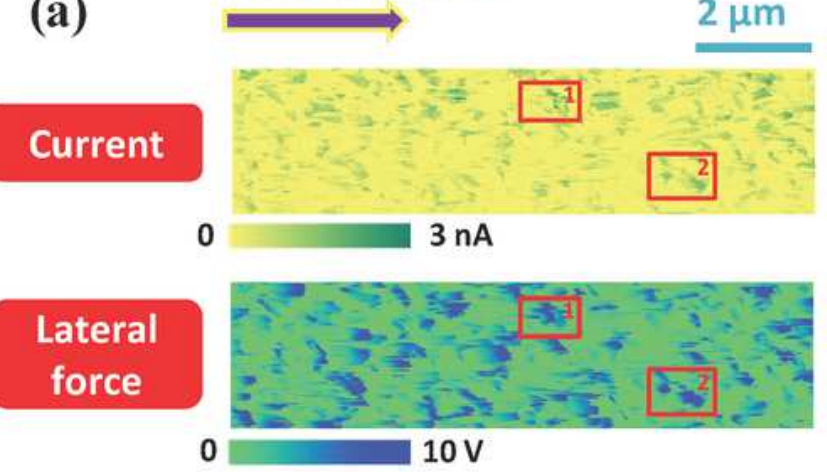

(b)

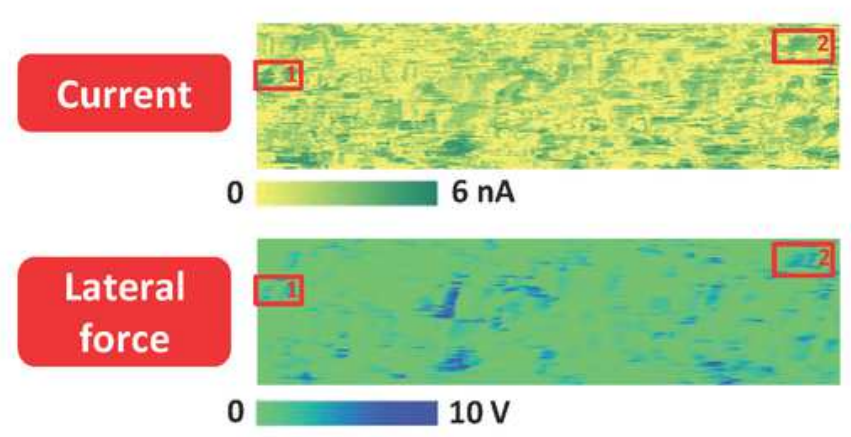

$$
\text { (a) }-1
$$
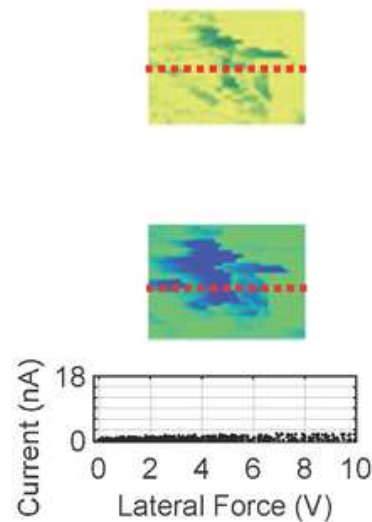

(b) -1
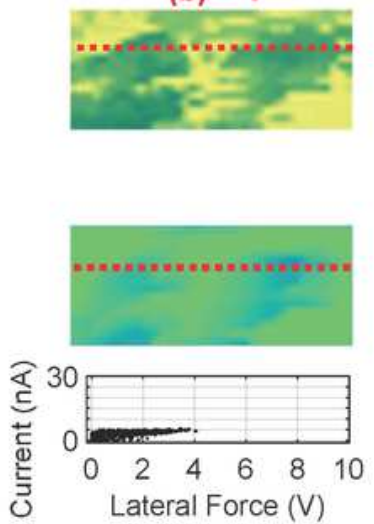

(a) -2
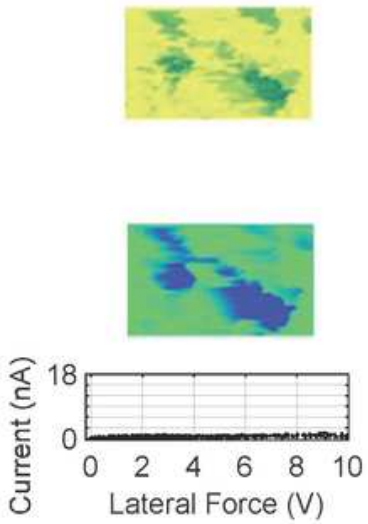

(b) -2
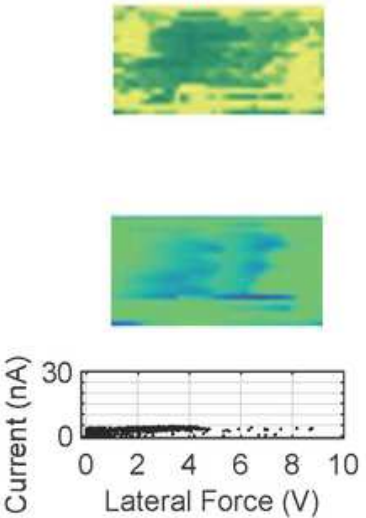

(c)

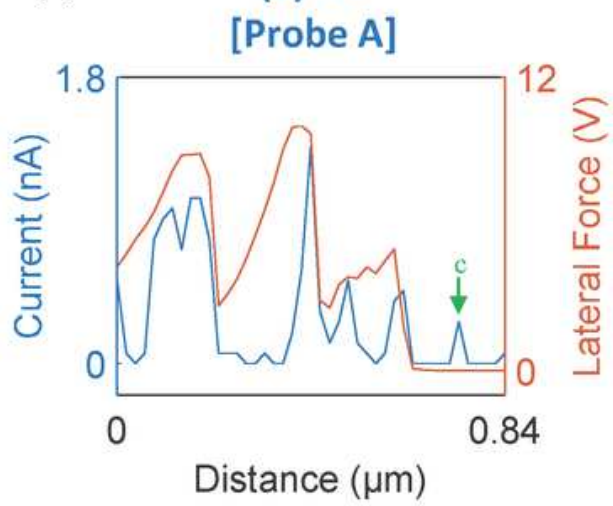

(b) -1

[Probe B]

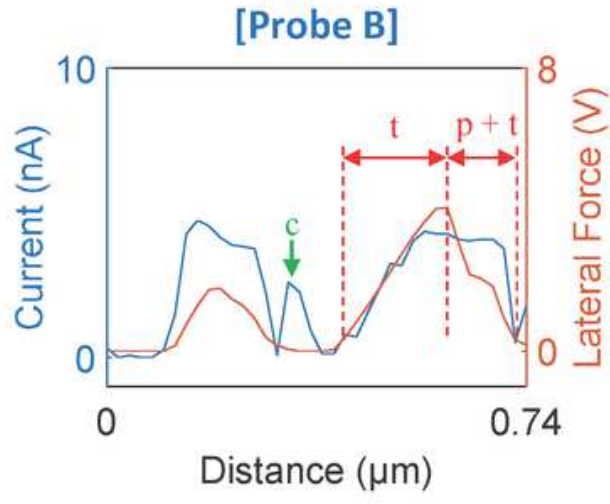

(d)

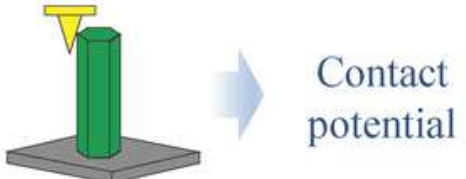

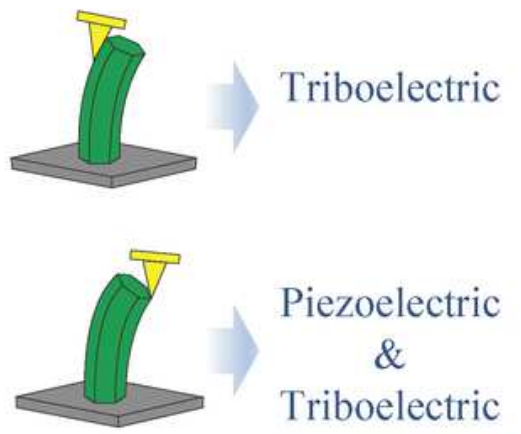

Figure 6

Pixel-wise comparison of the current and lateral force images. Pairs of current and lateral force images obtained using (a) Probe A and (b) Probe B during trace scans. Magnified images of the small areas marked by boxes are provided, with corresponding scatter plots on the right. (c) Line profiles of the magnified current and lateral force images in (a) and (b). (d) Schematics of three distinct current 
generation mechanisms: contact potential, triboelectric effect, and simultaneous piezoelectric and triboelectric effects 34 .

(a)

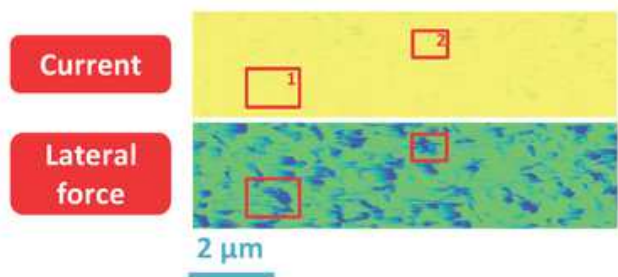

(b)

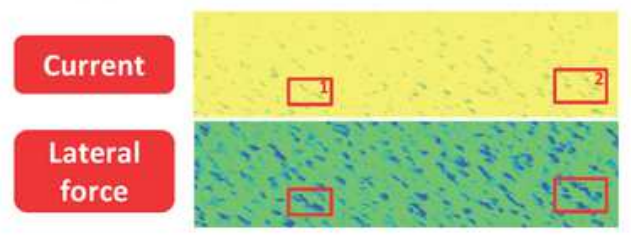

(c)

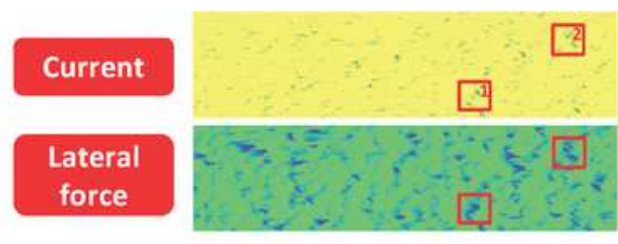

(d)

\section{Current}

Lateral

force

(e)

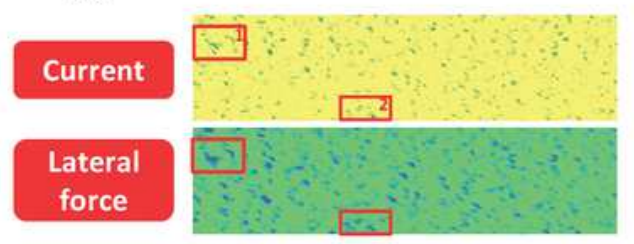

(a) -1

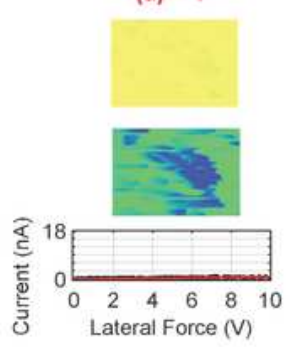

(b) -1

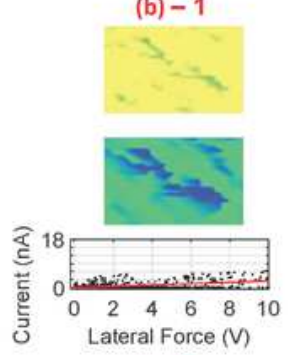

(c) -1

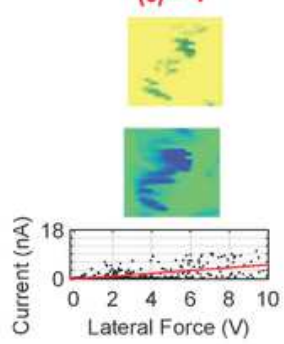

(d) -1

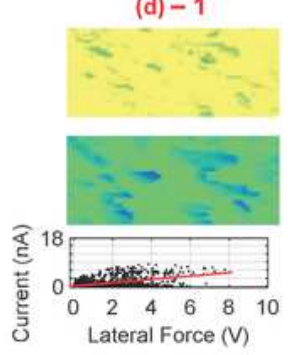

(e) -1

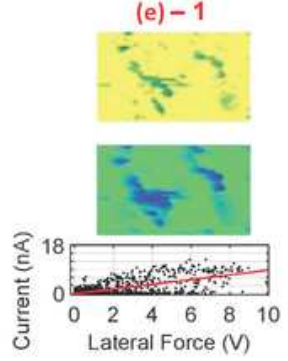

$10 \mathrm{~V}$

(a) -2

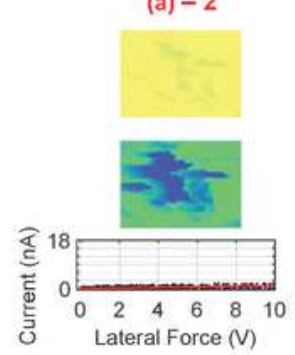

(b) -2

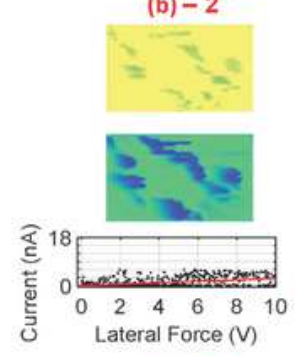

(c) -2

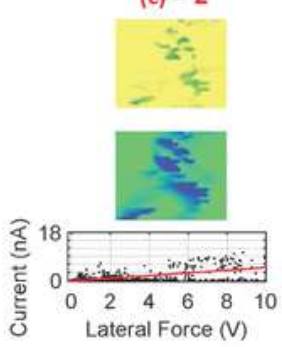

(d) -2

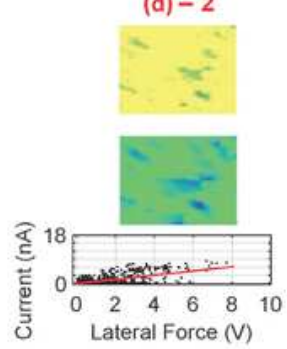

(e) -2

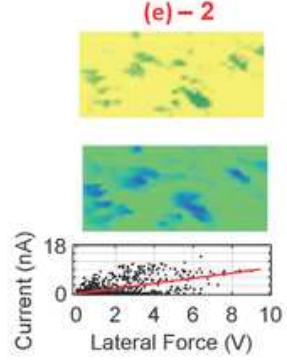

Figure 7

Variations in current and lateral force signals with the size of the $\mathrm{ZnO}$ nanorods. Pairs of current and lateral force images for (a) S6, (b) S12, (c) S18, (d) S24, and (e) S30 as measured by Probe A. In each 
figure part, magnified images of the areas marked by boxes and corresponding scatter plots are shown on the right. All images were obtained during trace scans.

(a)

Total current

Total lateral force

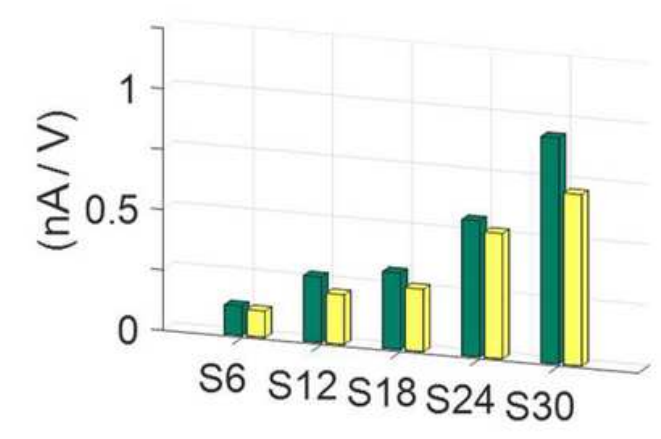

(b)

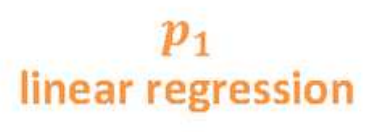

Probe A
Probe B
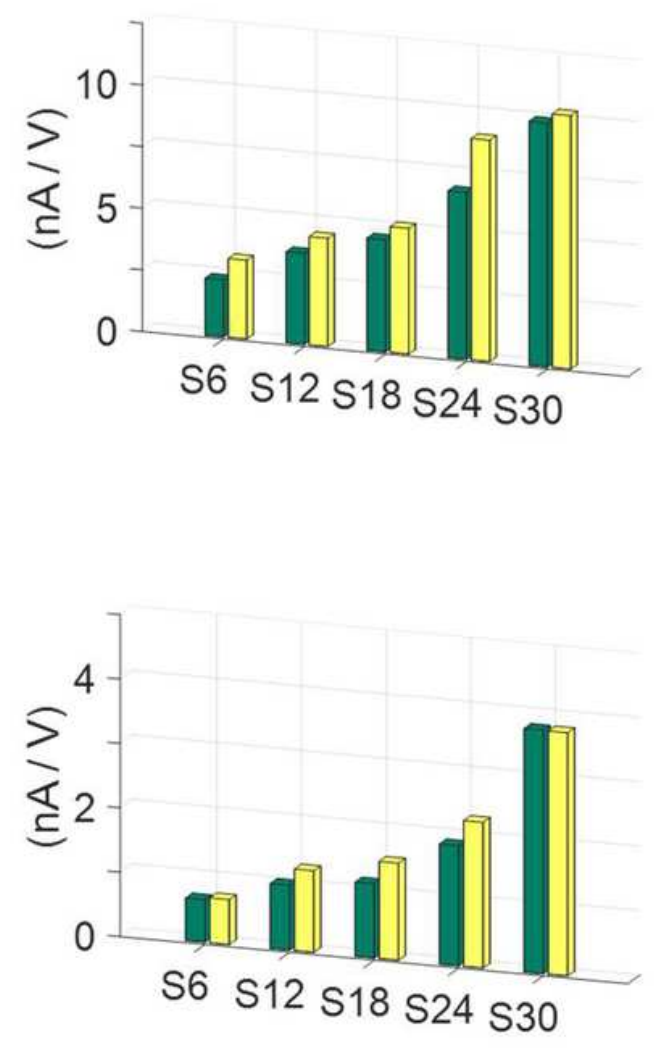

Retrace

\section{Figure 8}

Efficiency of conversion of the lateral force applied to the $\mathrm{ZnO}$ nanorods into current depending on the ZnO nanorod size. (a) Conversion efficiencies (total current/total lateral force) computed for each sample during trace and retrace scans using Probes A and B. (b) p_1 values for linear models (Eq. (1)) computed for each sample during trace and retrace scans by Probes $A$ and $B$.

\section{Supplementary Files}

This is a list of supplementary files associated with this preprint. Click to download.

- Supplementarylnformation.pdf 